\title{
Argininosuccinic aciduria: from a monogenic to a complex disorder
}

\author{
Ayelet Erez MD, $\mathrm{PhD}^{1,2}$
}

In the early 1930s, phenylketonuria was among the first metabolic diseases to be defined. In the following years, multiple attempts to correlate genotype and phenotype in several inherited metabolic diseases, including phenylketonuria, were encountered with difficulties. It is becoming evident that the phenotype of metabolic disorders is often more multifaceted than expected from the disruption of a specific enzyme function caused by a single-gene disorder. Undoubtedly, revealing the factors contributing to the discrepancy between the loss of a single enzymatic function and the wide spectrum of

Since the identification of phenylketonuria (PKU; OMIM 261600 ) in the early 1930s as a classical inborn error of metabolism (IEM), pioneering studies in PKU have revolutionized the diagnosis and management of IEM. ${ }^{1,2}$ PKU was the first genetic disorder to be diagnosed by newborn screen using the Guthrie tes $^{3,4}$ and was among the first disorders for which molecular testing by PCR was established. ${ }^{5}$ Most importantly, PKU was also the first IEM for which early treatment by dietary restriction of the substrate for the deficient enzyme effectively ameliorated disease manifestations. This finding specifically unraveled the importance of the interaction between the environment (dietary intake of phenylalanine) and the genotype. ${ }^{6}$ Yet despite the extensive studies on PKU, the correlation between the genotype and the neurological outcomes is still poor. This difficulty related to genotype-phenotype correlation is also observed in many other IEMs, and thus, again, PKU highlights the gaps in our understanding of the full spectrum of the pathogenicity of IEMs and points out the need for future studies on the topic. It is now evident that IEMs, despite being "monogenic disorders" at the molecular level, are truly "multifactorial disorders" at the phenotypic level. Importantly, it is also apparent that delineating the contributors to disease complexity will allow us to optimize therapy. ${ }^{7}$

Argininosuccinic aciduria (ASA; OMIM 207900), the second most common urea-cycle disorder, is another prototype of a complex IEM. ${ }^{8}$ ASA is caused by the deficiency of the urea cycle enzyme argininosuccinate lyase (ASL) that catalyzes the conversion of argininosuccinate to arginine and fumarate. The pathognomonic hallmark of ASA is the excretion of argininosuccinate in the urine. Because the secreted argininosuccinate is a nitrogen-rich compound, subjects with ASA typically tend to clinical consequences would allow clinicians to optimize treatment for their patients. This article discusses several possible contributors to the unique, complex phenotypes observed in inherited metabolic disorders, using argininosuccinic aciduria as a disease model.

Genet Med 2013:15(4):251-257

Key Words: arginine; argininosuccinate lyase; argininosuccinic aciduria; nitric oxide have fewer hyperammonemic episodes as compared with those with more proximal enzymatic blocks of ureagenesis, such as carbamoyl phosphate synthetase 1 and ornithine transcarbamylase deficiencies. ${ }^{9}$ Thus, the overall outcome in subjects with ASA would be expected to be better when compared with that of subjects with more proximal blocks of ureagenesis. However, despite fewer hyperammonemic episodes, studies have demonstrated that subjects with ASA have a greater risk for poor neurocognitive outcome,${ }^{10}$ hypertension, and liver disease. ${ }^{11-16}$ Even subjects with milder forms of ASA who are diagnosed early in life by newborn screens can develop neurocognitive deficiencies, attention deficit/hyperactivity disorder, developmental disability, and seizures despite early treatment intervention. ${ }^{17-19}$ Supporting this notion, hepatic disease and hypertension have been reported in patients with ASA who have good metabolic control. ${ }^{13,15,20-22}$

These data suggest that the complex phenotype observed in ASA involves mechanisms beyond the blockade in ureagenesis and that ASA could serve as an example of a complex IEM in which disease phenotype extends beyond the loss of the enzymatic function of ASL. The current therapeutic modalities used in the chronic treatment of ASA are dietary restriction and arginine supplementation, which are primarily geared toward the prevention of hyperammonemia. Further insights into the disease pathogenesis of ASA would allow optimization of treatment to prevent the long-term complications described.

In this article, ASA will be used as an example to demonstrate three unique aspects of IEMs that contribute to disease complexity: effects of metabolites on dynamic metabolic fluxes; participation of a single enzyme in multiple metabolic cycles; and structural requirements of the enzyme in addition to its 
catalytic function. Moreover, the article will illustrate how our evolving understanding of the complexity of ASA could benefit patients' outcome.

\section{EFFECTS OF METABOLITES ON DYNAMIC METABOLIC FLUXES}

Metabolites are the intermediates and products of metabolism, and the production or elimination of a quantity of metabolite per mass of organ or organism over a specific time frame is defined as metabolic flux. ${ }^{23}$ In contrast to the concept of a single-gene disorder causing disruption in a specific cellular pathway, metabolism involves a dynamic flux through multiple cycles that share metabolites, where outputs from one enzymatic reaction are inputs to other reactions. Hence, metabolism is the net entirety of a large network of all the metabolic reactions occurring simultaneously, "the metabolome." The use of isotopic labeling techniques has enabled us to study the metabolome, taking into account the kinetic measurements of time and the net effect of multiple metabolic pathways. ${ }^{24}$ Such sophisticated methods are required to deal adequately with environmental input while maintaining homeostasis, as well as with the complexities of eukaryotic organisms, which result from eukaryotes' multicellularity, compartmentalization of metabolites into organelles, and complex transport mechanisms. ${ }^{25-28}$

In the liver, ASL is one of the enzymes that participates in the urea cycle. A number of studies have attempted to draw a correlation between the genotype, which determines the level of ASL enzyme activity, and the phenotype severity of patients with ASA, with limited success. ${ }^{18}$ These difficulties suggest that the phenotype observed in ASA may result in part from the substrate accumulation (argininosuccinate) upstream of the enzymatic blockade, the deficiency of the metabolites downstream of the blockade, and their interactions with other metabolic cycles.

Recently, in a double-blind, placebo-controlled, crossover clinical study, we showed that subjects with ASA had higher levels of liver aminotransferases, when treated with a high dose of arginine as compared with lower dosage of arginine combined with sodium phenylbutarate, an ammonia scavenger. ${ }^{29}$ The increase in the plasma aminotransferases correlated with increases in argininosuccinate levels, suggesting that argininosuccinate may have a role in the hepatic injury described in ASA. In the high-dose arginine arm, there was an increase in urea flux as compared with the low-dose arginine arm, likely driving the cycle to produce higher levels of argininosuccinate. In parallel, the diversion of nitrogen away from the urea cycle in the low-dose arginine arm by sodium phenylbutyrate may have contributed to the decrease in urea flux and hence decreased the production of argininosuccinate (Figure 1), suggesting that such a treatment would be more suitable in preventing ASA liver injury.

Although the pathogenesis of hepatic disease in ASA is likely multifactorial, elevation of argininosuccinate is potentially an important factor. Our understanding of the alterations in metabolite concentrations and the dynamic metabolic fluxes by varying treatments suggests that administering a high dose of

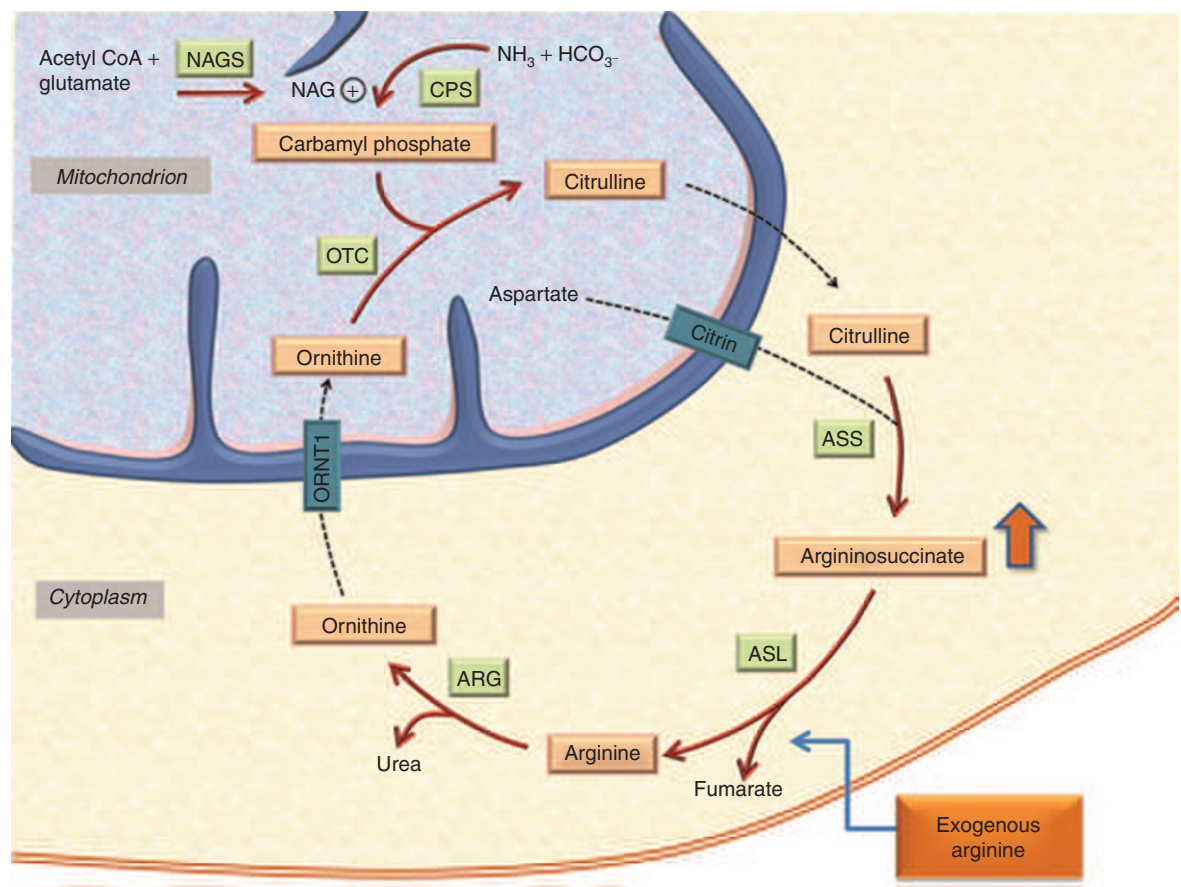

Figure 1 The urea cycle consists of six sequential enzymatic steps in which the nitrogen from ammonia and aspartate is transferred to urea. Deficiency of ASL leads to accumulation of argininosuccinate upstream of the block as well as deficiency of arginine downstream of the block. High-dose administration of exogenous arginine results in an increased production of argininosuccinate. ARG, arginase; ASL, argininosuccinate lyase; ASS, argininosuccinate synthetase; CPS1, carbamyl phosphate synthetase 1; NAG, N-acetylglutamate; ORNT1, ornithine transporter; OTC, ornithine transcarbamylase. Modified with permission from a review on ASA written for the AJMG, part C. 
arginine to subjects with ASA can result in abnormalities in the liver function tests, and therefore lower arginine dose together with sodium phenylbutarate might be more appropriate.

In addition to the possible toxic effects caused by the accumulation of the upstream metabolites in ASL deficiency, the deficiency of arginine and fumarate, the metabolites synthesized by ASL, could have further consequences. Arginine is synthesized endogenously only by ASL and is the substrate for the generation of multiple metabolites, including nitric oxide (NO), polyamines, proline, creatine, glutamate, agmatine, and urea (see Supplementary Figure S1 online). All these metabolites have established roles in multiple metabolic pathways involved in cell signaling (NO, agmatine), survival (NO), and proliferation (polyamines, proline). In addition, fumarate is an important intermediate in the citric acid cycle for energy generation. It is thus reasonable to hypothesize that ASL deficiency could have additional consequences on pathways that depend on the substrates generated by ASL.

In contrast to arginine, which is endogenously synthesized only by ASL, fumarate may be generated by other enzymes. Nevertheless, the urea cycle and Krebs cycle are linked to each other at the cross point of ASL, where aspartate from the Krebs cycle can enter the urea cycle to generate argininosuccinate that is converted to arginine and fumarate by ASL and fumarate could return to the Krebs cycle. ${ }^{30}$ Thus, it is possible that ASL deficiency might also affect the Krebs cycle and therefore the cellular energy metabolism (Figure 2).

\section{PARTICIPATION OF A SINGLE ENZYME IN MULTIPLE METABOLIC CYCLES}

Participation of an enzyme in different metabolic cycles could also contribute to the complexity of metabolic disorders. The term "moonlighting" was coined by Jeffery in 1999 to describe the phenomenon in which several metabolic enzymes exhibit multiple functions potentially involving different metabolic cycles. The presence of a protein in an unexpected location in the cell, in an unexpected cell type, or in an unexpected multiprotein complex could point to possible moonlighting activities.

Although ASL and argininosuccinate synthase (ASS1) are part of the urea cycle in the liver, they are also expressed in most tissues outside the liver. It is possible that their expression in various tissues is driven by the arginine synthesis requirement for other metabolic cycles. For example, NO synthesis uses arginine as a substrate. ASL and ASS1 are key players in the citrulline-NO cycle, in which citrulline is regenerated from arginine for the synthesis of NO. Using stable-isotope labeling of the guanidino group of arginine, we were able to show that subjects with ASA had decreased fractional transfer of the guanidino nitrogen from ${ }^{15} \mathrm{~N}_{2}$-arginine to ${ }^{15} \mathrm{~N}$-citrulline, reflecting decreased NO production. This was despite the fact that subjects with ASA had higher arginine flux and higher plasma concentrations of arginine as compared with the controls. ${ }^{14}$ Corroborating these human studies, liver-targeted gene therapy with Asl in mice with ASA resulted in correction of the hyperammonemia and survival, but these mice still demonstrated reduced fractional transfer of guanidino nitrogen from ${ }^{15} \mathrm{~N}_{2}$-arginine to ${ }^{15} \mathrm{~N}$-citrulline, indicating decreased systemic $\mathrm{NO}$ production. Furthermore, NO production in the aortic vessels of these mice was impaired, elucidating the occurrence of hypertension. Thus, the deficiency of ASL results in decreased NO production at the level of the whole organism and in multiple tissues, signifying the requirement of ASL for different metabolic reactions performed by different enzymes such as nitric oxide synthase (NOS) (Figure 3). Unraveling the contribution of NO deficiency to the pathogenesis of ASA paved the way to studying the beneficial effects of implementing $\mathrm{NO}$ supplementation as additional therapy in ASA.

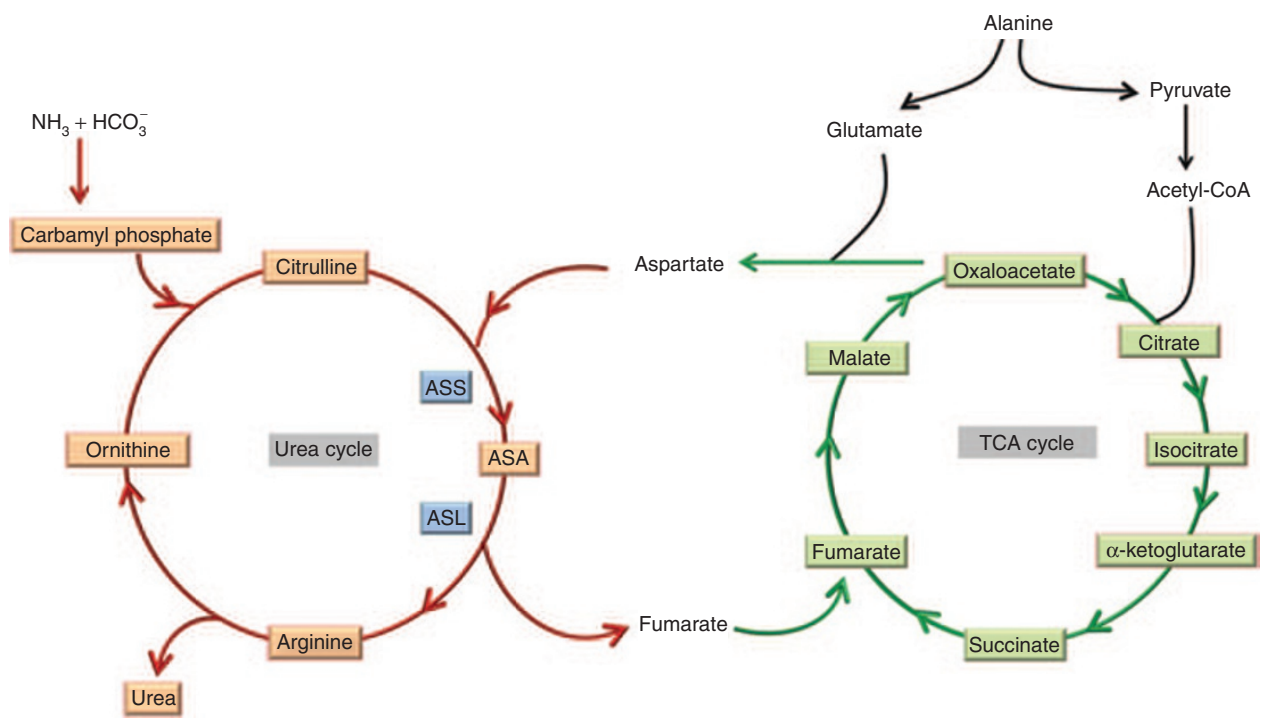

Figure 2 The urea and Krebs cycle. The urea cycle is shown on the left and the Krebs cycle on the right. Alanine, as a prototype amino acid, is transaminated to pyruvate that is decarboxylated to enter the Krebs cycle and gives up its amino group to ketoglutarate for the formation of glutamate. The glutamate transaminates to aspartate that enters the urea cycle to form argininosuccinate (ASA), which will be cleaved to arginine and fumarate. The latter re-enters the Krebs cycle, whereas arginine is available for further metabolism. ASL, argininosuccinate lyase; ASS, argininosuccinate synthetase; TCA, tricarboxylic acid cycle. 

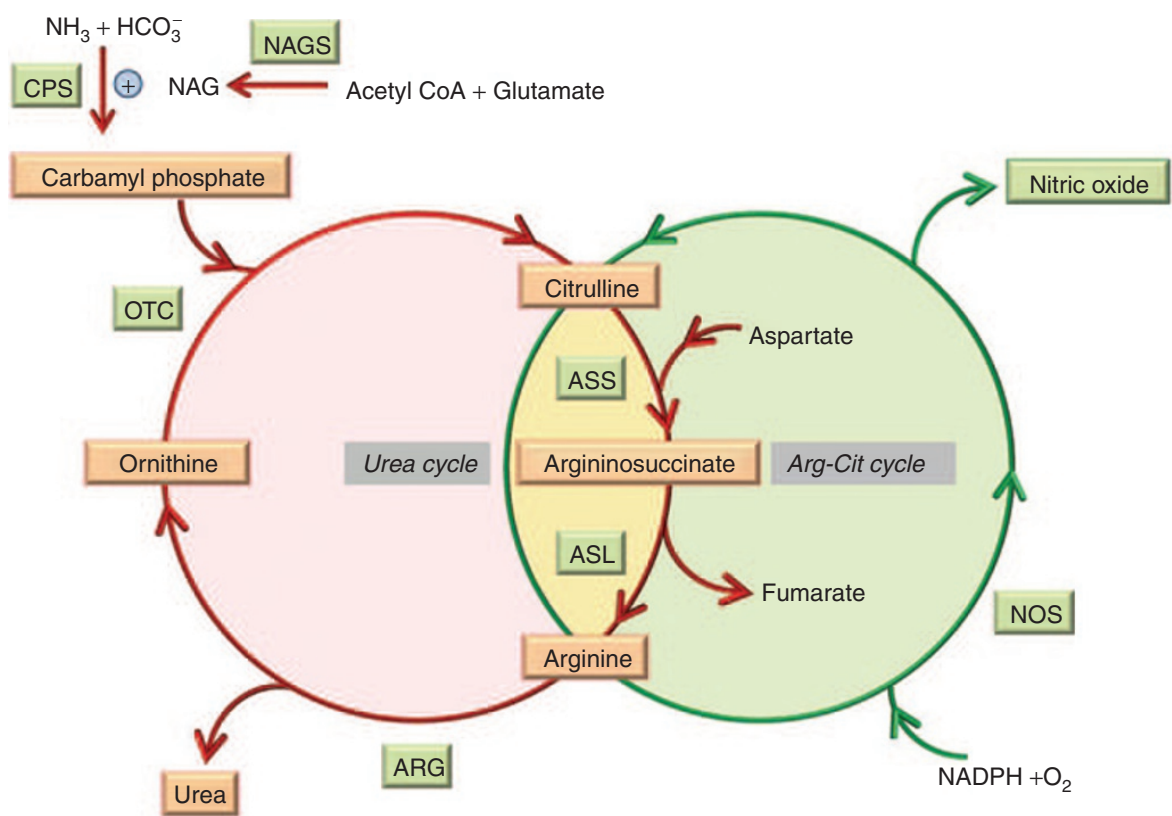

Figure 3 The urea cycle and the arginine-citrulline cycle. Although ASL is involved in the urea cycle with no net synthesis of arginine in the liver, many tissues depend on it for the regeneration of arginine. In most tissues, the arginine-citrulline cycle couples arginine recycling to citrulline by nitric oxide synthase (NOS), with the generation of nitric oxide (NO). ARG, arginase; ASL, argininosuccinate lyase; ASS, argininosuccinate synthetase; CPS, carbamyl phosphate synthetase; NADPH, nicotinamide adenine dinucleotide phosphate-oxidase; NAG, N-acetylglutamate; NAGS, N-acetylglutamatesynthetase; OTC, ornithine transcarbamylase. Taken with permission from a review on ASA written for AJMG, part C.

As a secondary effect, the lack of arginine substrate availability for NOS results in the "uncoupling" of NOS, in which free radicals are generated instead of NO. ${ }^{31-33}$ Mice with ASA indeed show elevation of free radicals in the vessels as well as in plasma and urine. ${ }^{14,15}$ Increased free radical production could add to the tissue damage, contributing to the vessels and liver injury. ${ }^{34}$ In addition, in the context of ASA, it may be particularly damaging because argininosuccinate could be converted to guanidinosuccinic acid, a known cellular and neuronal toxin. It is therefore possible that the neurocognitive delays observed in ASA could partly result from the increased generation of guanidinosuccinic acid. ${ }^{35-37}$

Although the decreased NO production as a consequence of ASL deficiency was only shown for NO as a direct product of arginine, it is possible that other products of arginine, such as polyamines, proline, creatine, glutamate, and agmatine, are affected as well. Further studies are needed to determine whether free radicals as well as all other arginine metabolites contribute to the cognitive delay, liver cirrhosis, and hypertension in patients with ASA.

\section{STRUCTURAL REQUIREMENTS}

The active sites in multiple enzymes usually do not occupy a large fraction of the protein surface area, leaving considerable solvent-exposed surface that can be recruited for other moonlighting functions. It is therefore likely that additional activities of several proteins have evolved to make use of such "unused sites" on the protein surface. ${ }^{38}$ Furthermore, a change in the cellular localization of a protein or in its folding will expose

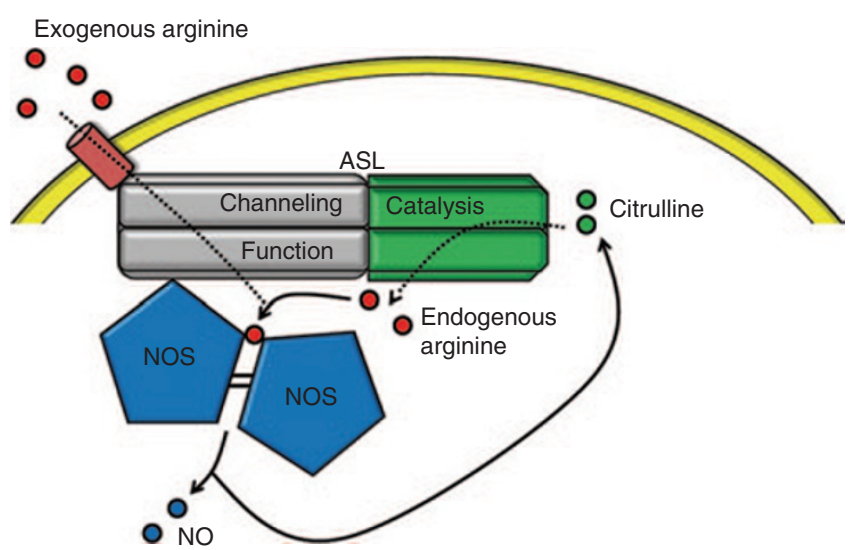

Figure 4 The catalytic and structural functions of ASL. ASL is not only required for endogenous arginine synthesis (a catalytic function) but is also structurally required to maintain a complex that facilitates channeling of exogenous arginine to nitric oxide synthase (NOS) for nitric oxide (NO) production. The tetrameric ASL enzyme depicted in green represents the region that is required for catalysis, whereas the region in gray is needed for the NO synthesis complex that includes SCL7A1, the cationic arginine transporter, and ASS1. ASL, argininosuccinate lyase. Taken with permission from a review on ASA therapy written for MGM, 2012.

different regions of its surface and may cause the protein to form complexes with different proteins, altering their functional role. ${ }^{39}$ The dual regulation of the structural and catalytic functions of the protein may be influenced by the cellular concentration of the ligand, complex proteins, substrates, cofactors, or product availabilities. ${ }^{40}$ 
a

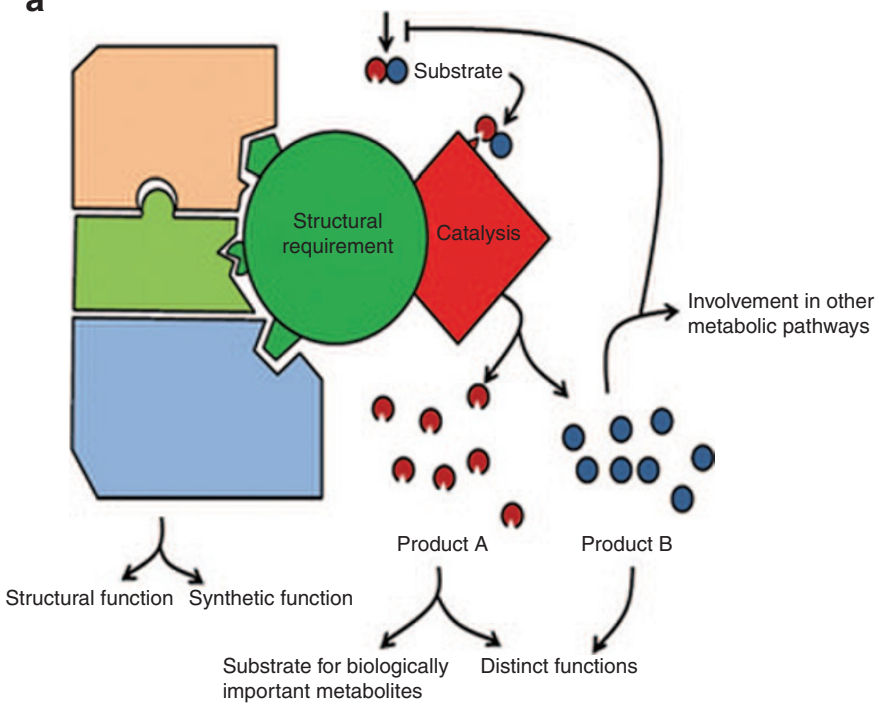

b

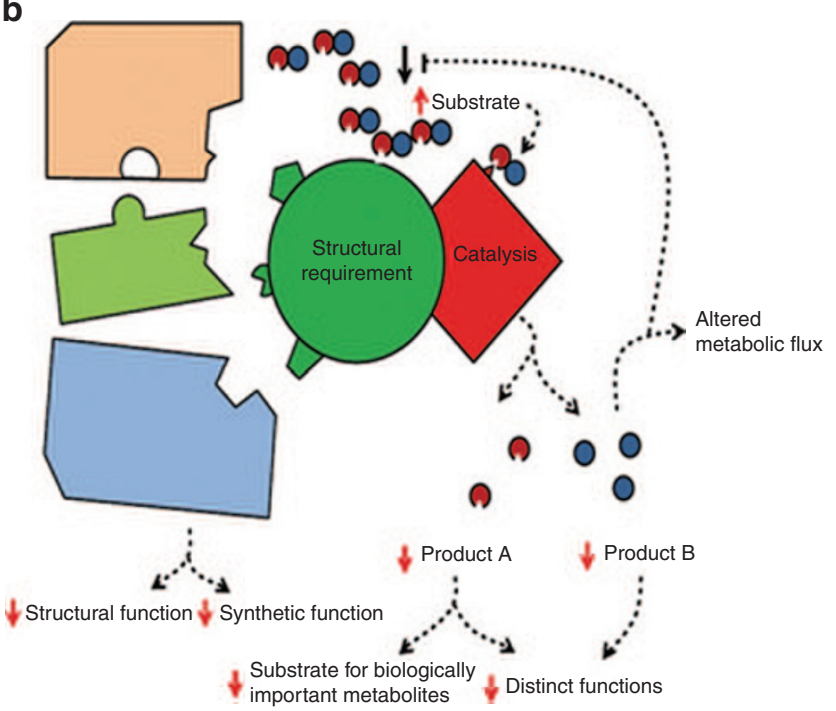

Figure 5 The possible metabolic consequences of different mutations in one enzyme. (a) Under normal conditions, the enzyme potentially contributes to product synthesis, has a structural requirement for forming complexes with other proteins, and is part of the net metabolic flux through multiple cycles. (b) Different mutations can affect the enzyme catalytic and/or structural functions and alter the net metabolic flux.

ASL belongs to a superfamily of metabolic enzymes that includes fumarase, aspartase, $\delta$ crystallin, adenylosuccinate lyase, and 3-carboxy-cis, cis-muconate lactonizing enzyme. ${ }^{41}$ These enzymes function as tetramers and catalyze reactions in which fumarate is generated as a product. Among all the enzymes in this family, $\delta 2$ crystallin, a protein present in the avian and reptilian eye lens, has a high degree of homology to ASL. ${ }^{42-44} \delta 2$ Crystallin has structural functions that are necessary for the maintenance of transparency of the lens and are distinct from its catalytic function, which, similar to ASL, is the synthesis of arginine. ${ }^{45}$

Whereas the enzymatic properties of ASL have been well characterized, we recently showed an additional structural requirement of ASL for the assembly of a NO synthesis complex. Using the ASA mouse model and human ASL-deficient cells, we found that ASL is required for directing exogenous arginine to a complex necessary for the synthesis of NO. ${ }^{14}$ Despite sufficient levels of arginine, the substrate for NOS, loss of ASL resulted in decreased abundance of a NOS complex, leading to decreased NO production. The deficiency of NO production could be rescued by transfection with catalytically inactive forms of ASL, thus confirming the dependence of the NOS complex assembly on the structural integrity, but not the enzymatic function, of ASL. Our studies distinguished between two essential roles of ASL: the catalytic function required for ureagenesis in the liver and endogenous arginine synthesis in the rest of the body; and the maintenance of a NOS complex required for efficient $\mathrm{NO}$ production from extracellular sources of arginine (Figure 4).

In addition, these findings contribute to our understanding of the poor correlation between enzymatic activity and clinical outcome in $\mathrm{ASA}^{11,12}$ and have important clinical implications for therapy. ${ }^{15,29}$ Because ASL deficiency is an autosomal recessive disorder, patients typically have compound heterozygous mutations, and therefore it is hard to predict their response to arginine treatment. Hence mutations in ASL can have different effects on the catalytic versus structural properties of the enzyme, and it is thus likely that some patients would have minimal deficiencies in NO production, whereas the deficiencies in others may be significantly greater. Moreover, nutritional intake of NO sources may further modify the clinical manifestation of any potential $\mathrm{NO}$ deficiency, emphasizing again the important effect of the environment on clinical phenotype.

As a proof of concept, we initiated treatment with NO supplementation in one of the subjects with ASA under our clinical care who had chronic resistant hypertension. Treatment with NOS-independent NO donor in this patient resulted in sustained normalization of blood pressure, permitting discontinuation of all other antihypertensive medications. Anecdotally, improvements in several of his neuropsychological parameters pertaining to verbal memory and nonverbal problem solving were also noted with the NO therapy. ${ }^{15,29}$ Although these human data are preliminary and call for a clinical trial, they imply that NO supplementation in ASA may be beneficial, and therefore, higher intake of nitrite-rich foods may be of benefit in patients with ASA.

\section{DISCUSSION}

Although IEMs are inherited in a simple Mendelian manner, their phenotypes appear to be inherited as complex traits. ${ }^{28}$ This article uses ASA as an example of the complexity of metabolic diseases to illustrate how gaining insights into disease pathogenicity could benefit patients.

Because ASL is the only catalytic enzyme responsible for endogenous arginine generation, the most significant consequence of ASL deficiency is in the liver, where its malfunction 
in the urea cycle causes hyperammonemia. The secondary manifestations are the accumulation of ASL upstream metabolite argininosuccinate and the deficiency of arginine and its downstream metabolites, such as NO. Thus, the conventional long-term treatment for ASA intended to provide metabolic control and prevent hyperammonemia is arginine supplementation and dietary restriction. However, recent studies show that simple replenishment of arginine is not as effective in preventing the long-term disease complications such as hypertension, liver fibrosis, and neurocognitive delay. Dynamic metabolic flux studies showed that high doses of arginine can result in increased production of argininosuccinate that might contribute to liver damage. In addition, metabolic studies revealed that with ASL deficiency, there is a consequent deficiency of NO because it is one of the metabolites generated from arginine. Importantly, combining metabolic and proteomic studies, we showed that exogenous arginine does not replete the production of NO because of the structural requirement of ASL for the assembly of the NO synthesis complex. Other downstream metabolites of arginine are also possibly dysregulated with ASL deficiency, potentially leading to further cellular injuries, and thus, there are still open questions related to the participation of ASL in other cycles. Therefore, additional studies are needed to dissect the contribution of each to clinical manifestations of ASA.

Specifically for ASA, the recent discoveries reviewed here suggest treatment modification with lower arginine dose together with sodium phenylbutarate and dietary NO supplementation could benefit patients. More generally for IEMs, these studies emphasize the multifactorial aspects of metabolic diseases and the importance of understanding the integration of the genome-transcriptome-proteome-metabolome with the environment for optimizing treatment (Figure 5).

\section{SUPPLEMENTARY MATERIAL}

Supplementary material is linked to the online version of the paper at http://www.nature.com/gim

\section{ACKNOWLEDGMENTS}

This work was supported by National Institutes of Health grant K08 DK081735. The author thanks her mentor Prof Brendan Lee for his vision, guidance, and endless support; Sandesh C.S. Nagamani, a colleague and friend, for his valuable edits and illustrations; and the William K. Bowes, Jr. Award Committee for their generosity, recognition, and promotion of medical geneticists.

\section{DISCLOSURE}

The author declares no conflict of interest.

\section{REFERENCES}

1. Scriver CR, Clow CL. Phenylketonuria and other phenylalanine hydroxylation mutants in man. Annu Rev Genet 1980;14:179-202.

2. Woo SL, Lidsky AS, Güttler F, Chandra T, Robson KJ. Cloned human phenylalanine hydroxylase gene allows prenatal diagnosis and carrier detection of classical phenylketonuria. Nature 1983;306:151-155.

3. Guthrie R, Susi A. A simple phenylalanine method for detecting phenylketonuria in large populations of newborn infants. Pediatrics 1963;32:338-343.

4. Lindee MS. Genetic disease since 1945. Nat Rev Genet 2000;1(3):236-241.
5. DiLella AG, Huang WM, Woo SL. Screening for phenylketonuria mutations by DNA amplification with the polymerase chain reaction. Lancet 1988;1(8584):497-499.

6. Scriver $C R$, Waters PJ. Monogenic traits are not simple: lessons from phenylketonuria. Trends Genet 1999;15:267-272.

7. Berry GT, Elsas LJ. Introduction to the Maastricht workshop: lessons from the past and new directions in galactosemia. J Inherit Metab Dis 2011;34:249-255.

8. Engel K, Vuissoz JM, Eggimann S, et al. Bacterial expression of mutant argininosuccinate lyase reveals imperfect correlation of in-vitro enzyme activity with clinical phenotype in argininosuccinic aciduria. J Inherit Metab Dis 2012:35:133-140.

9. Batshaw ML. Hyperammonemia. Curr Probl Pediatr 1984;14:1-69.

10. Ah Mew N, Krivitzky L, McCarter R, et al. Clinical outcomes of neonatal onset proximal versus distal urea cycle disorders do not differ. J Pediatr 2012; e-pub ahead of print 14 August 2012.

11. Erez A, Nagamani SC, Lee B. Argininosuccinate lyase deficiency-argininosuccinic aciduria and beyond. Am J Med Genet C Semin Med Genet 2011;157: 45-53.

12. Nagamani SCS, Erez A, Lee B. Argininosuccinate lyase deficiency. In: Pagon RA, Bird TD, Dolan CR, Stephens K, Adam MP (eds). GeneReviews 2011.

13. Brunetti-Pierri N, Erez A, Shchelochkov O, Craigen W, Lee B. Systemic hypertension in two patients with ASL deficiency: a result of nitric oxide deficiency? Mol Genet Metab 2009;98:195-197.

14. Erez A, Nagamani SC, Shchelochkov OA, et al. Requirement of argininosuccinate lyase for systemic nitric oxide production. Nat Med 2011;17:1619-1626.

15. Nagamani SC, Campeau PM, Shchelochkov OA, et al. Nitric-oxide supplementation for treatment of long-term complications in argininosuccinic aciduria. Am J Hum Genet 2012;90:836-846.

16. Nagamani SC, Erez A, Lee B. Argininosuccinate lyase deficiency. Genet Med 2012;14:501-507.

17. Tuchman M, Lee B, Lichter-Konecki U, et al. Cross-sectional multicenter study of patients with urea cycle disorders in the United States. Mol Genet Metab 2008:94:397-402.

18. Ficicioglu C, Mandell R, Shih VE. Argininosuccinate lyase deficiency: long-term outcome of 13 patients detected by newborn screening. Mol Genet Metab 2009;98:273-277.

19. Mercimek-Mahmutoglu S, Moeslinger D, Häberle J, et al. Long-term outcome of patients with argininosuccinate lyase deficiency diagnosed by newborn screening in Austria. Mol Genet Metab 2010;100:24-28.

20. Mori T, Nagai K, Mori M, et al. Progressive liver fibrosis in late-onset argininosuccinate lyase deficiency. Pediatr Dev Pathol 2002;5:597-601.

21. Billmeier GJ Jr, Molinary SV, Wilroy RS Jr, Duenas DA, Brannon ME. Argininosuccinic aciduria: investigation of an affected family. J Pediatr 1974;84: 85-89.

22. Zimmermann A, Bachmann C, Baumgartner R. Severe liver fibrosis in argininosuccinic aciduria. Arch Pathol Lab Med 1986;1 10:136-140.

23. Lanpher B, Brunetti-Pierri N, Lee B. Inborn errors of metabolism: the flux from Mendelian to complex diseases. Nat Rev Genet 2006;7:449-460.

24. Kelleher JK. Flux estimation using isotopic tracers: common ground for metabolic physiology and metabolic engineering. Metab Eng 2001;3:100-110.

25. Schryer DW, Peterson P, Paalme T, Vendelin M. Bidirectionality and compartmentation of metabolic fluxes are revealed in the dynamics of isotopomer networks. Int J Mol Sci 2009;10:1697-1718.

26. Rahib L, MacLennan NK, Horvath S, Liao JC, Dipple KM. Glycerol kinase deficiency alters expression of genes involved in lipid metabolism, carbohydrate metabolism, and insulin signaling. Eur J Hum Genet 2007;15:646-657.

27. Dipple KM, Phelan JK, McCabe ER. Consequences of complexity within biological networks: robustness and health, or vulnerability and disease. Mol Genet Metab 2001;74:45-50.

28. Dipple KM, McCabe ER. Phenotypes of patients with "simple" Mendelian disorders are complex traits: thresholds, modifiers, and systems dynamics. Am J Hum Genet 2000;66:1729-1735.

29. Nagamani SC, Lee B, Erez A. Optimizing therapy for argininosuccinic aciduria. Mol Genet Metab 2012; ; 107:10-14.

30. Shambaugh GE 3rd. Urea biosynthesis I. The urea cycle and relationships to the citric acid cycle. Am J Clin Nutr 1977;30:2083-2087.

31. Pignitter M, Gorren AC, Nedeianu S, Schmidt K, Mayer B. Inefficient spin trapping of superoxide in the presence of nitric-oxide: implications for studies on nitric-oxide synthase uncoupling. Free Radic Bio/ Med 2006;41:455-463.

32. Lin MI, Fulton D, Babbitt R, et al. Phosphorylation of threonine 497 in endothelial nitric-oxide synthase coordinates the coupling of $\mathrm{L}$-arginine metabolism to efficient nitric oxide production. J Biol Chem 2003;278:44719-44726. 
33. Stuehr D, Pou S, Rosen GM. Oxygen reduction by nitric-oxide synthases. J Biol Chem 2001;276:14533-14536.

34. Halliwell B. Reactive oxygen species and the central nervous system. J Neurochem 1992;59:1609-1623.

35. Aoyagi K. Inhibition of arginine synthesis by urea: a mechanism for arginine deficiency in renal failure which leads to increased hydroxyl radical generation. Mol Cell Biochem 2003;244:11-15.

36. Aoyagi K, Shahrzad S, lida S, et al. Role of nitric oxide in the synthesis of guanidinosuccinic acid, an activator of the N-methyl-D-aspartate receptor. Kidney Int Supp/ 2001;78:S93-S96.

37. D'Hooge R, Pei YQ, Marescau B, De Deyn PP. Convulsive action and toxicity of uremic guanidino compounds: behavioral assessment and relation to brain concentration in adult mice. J Neurol Sci 1992;112:96-105.

38. Jeffery CJ. Moonlighting proteins. Trends Biochem Sci 1999;24:8-11.

39. Andreeva A, Murzin AG. Evolution of protein fold in the presence of functional constraints. Curr Opin Struct Biol 2006;16:399-408.
40. Jeffery CJ. Moonlighting proteins: old proteins learning new tricks. Trends Genet 2003;19:415-417.

41. Turner MA, Simpson A, Mclnnes RR, Howell PL. Human argininosuccinate lyase: a structural basis for intragenic complementation. Proc Natl Acad Sci USA 1997;94:9063-9068.

42. Yeh LS, Elzanowski A, Hunt LT, Barker WC. Homology of delta crystallin and argininosuccinate lyase. Comp Biochem Physiol, B 1988;89: 433-437.

43. Wistow G, Piatigorsky J. Recruitment of enzymes as lens structural proteins. Science 1987;236:1554-1556.

44. Vallée F, Turner MA, Lindley PL, Howell PL. Crystal structure of an inactive duck delta II crystallin mutant with bound argininosuccinate. Biochemistry 1999;38:2425-2434.

45. Lee HJ, Chiou SH, Chang GG. Biochemical characterization and kinetic analysis of duck delta-crystallin with endogenous argininosuccinate lyase activity. Biochem J 1992;283(Pt 2):597-603. 